\title{
AIR TEMPERATURE EXPOSURE AND AGRICULTURAL OCCUPATIONAL INJURIES IN THE AUTONOMOUS PROVINCE OF TRENTO (2000-2013, NORTH-EASTERN ITALY)
}

\section{MATTEO RICCò}

Provincial Agency for Health Services of the Autonomous Province of Trento (APSS), Trento, Italy

Department of Prevention, Health and Safety Unit

\begin{abstract}
Objectives: The aim of this study was to evaluate the relationship between high air temperatures and occupational injuries (OIs) occurred during the summer seasons 2000-2013 in agricultural workers from the Autonomous Province of Trento (APT), North-Eastern Italy. Material and Methods: Data about OIs for the APT from 2000 to 2013 occurring during the warm season $(\mathrm{N}=7325)$ was provided by the National Institute of Insurance for Occupational Illness and Injury. Daily average and daily maximum temperatures values for the specific geographical site of events were retrieved. Daily temperatures were then assessed in 3 time lags: for the day of the event (lag 0), and for the previous $24 \mathrm{~h}$ (lag 1) and $48 \mathrm{~h}$ (lag 2). Daily temperatures were then categorized in 3 exposure groups $(<75$ th, $75-95$ th and $>95$ th percentiles). The risk of OIs was assessed as odds ratio (OR) calculated through a Poisson regression model controlled for age, sex, ethnicity and time period, and assuming OI rates for days on which temperature was comprised in < 75th percentile exposure groups as the referent ones. Results: Estimated incidence of OIs during the study period was $3.4 \pm 2.3$ events/day. The peak of work-related accidents occurred on days characterized by severe thermal conditions, and in particular during heat waves (incidence rate ratio $=1.09,95 \%$ confidence interval $(\mathrm{CI}): 1.02-1.17, \mathrm{p}=0.0165$ ). Days having temperatures higher than 95th percentile, assessed as daily average, both on current days (OR $=1.119,95 \%$ CI: $1.008-1.242)$ and in lag $1(\mathrm{OR}=1.125,95 \% \mathrm{CI}$ : 1.013-1.249), as well as daily maximum temperatures, were at the highest risk of work-related injuries $(\mathrm{OR}=1.144,95 \% \mathrm{CI}$ : 1.029-1.272). Conclusions: In conclusion, presented findings recommend policymakers to develop appropriate warning/alert systems for agricultural workers regarding high environmental temperatures. Int J Occup Med Environ Health 2018;31(3):317-331
\end{abstract}

Key words:

Agricultural workers, Climate change, Heat exposure, Occupational injuries, Hot weather, Heat wave

\section{INTRODUCTION}

During the last decades, average global surface temperatures have steadily increased, with more hot days and warm nights, fewer cool days and cold nights, and more frequent, hotter and longer heatwaves (HWs) [1-3]. Available estimates suggest that the increased magnitude and frequency of extreme events would ultimately lead to a significant excess in morbidity and mortality for cardiovascular and respiratory illnesses, and that the climate changes will presumptively increase both the incidence and the severity of these effects [4-12], in particular in Mediterranean-like climates [13].

Received: August 28, 2016. Accepted: June 8, 2017.

Corresponding author: M. Riccò, Provincial Agency for Health Services of the Autonomous Province of Trento (APSS), Department of Prevention, Health and Safety Unit, Viale Verona SNC C/O Big Center, 38123 Trento, Italy (e-mail: mricco2000@gmail.com, matteo.ricco@apss.tn.it). 
In certain occupational settings, heat exposure would represent an even greater challenge $[5,14]$ because of a combination of external thermal environment, heat sources in the workplace, and internal heat generation by strenuous physical activity through muscular work [15-17]. Evidence suggests that the exposure to high environmental temperatures not only affects workers' capability to undertake physical activities without harm, especially in subjects with pre-existing illnesses [2,5,18-24] but high temperatures would significantly hamper cognition and concentration of the workers, reducing vigilance and increasing fatigue: as a consequence, working during warm weather would also ultimately increase the risk of mistakes, accidents and occupational injuries (OIs) [14,25-29].

The risk of heat-related health effects appears to be significantly increased in outdoors workers such as agricultural workers (AWs), for several reasons [5,30,31]. First of all, many agricultural activities are performed during the warm season, often requiring the use of personal protective equipment (PPE). As the wearing of PPEs increases physical strain and restricts heat loss from the body, it directly increases the risk for heat-related illness [3,5,22,32], at the same time prompting some workers to reduce their use, ultimately increasing the risk for incidents associated with the use of chemicals, such as pesticides and fertilizers [28].

Secondly, even though the mechanization of many agricultural activities has restrained the strenuous physical labor carried out by AWs, therefore reducing their vulnerability to environmental and exertional heath stress, many farming activities still requires manual work, also in high income countries (e.g., collection of apples and oranges). Unfortunately, extensive mechanization requires economic resources and access to a suitable energy supply, being often beyond the financial capacity of most agricultural communities in low income countries, and also for some small-sized agricultural enterprises in high income countries [17]. Thirdly, health care and safety training in AWs are frequently inadequate, in particular among subjects with migrant background [33,34], part-time or seasonal workers, and "hobby farmers" (i.e., subjects running a farm as a hobby rather than as means of making a living), the number of which usually surges during hot moths and the harvest season, including subjects who otherwise spend little time outdoor $[25,29,35]$.

Hence, many AWs may continue to work beyond a safe heat exposure limit as they are unaware of the risks associated with the heat exposure or have inappropriate knowledge of the preventive measures, as avoiding the hottest hours of the day for most strenuous physical exertion or increasing the water intake, in particular during the HWs $[13,30]$. As climate change effects gradually progress, the importance of understanding the impact of hot climate on injuries in AWs and preventing them through appropriate preparedness and emergency response plans in the workplace has become a growing challenge for occupational health and safety $[2,16,28,36]$. However, despite $4.6 \%$ of the total European Union workforce is still engaged in the agricultural sector, little data is available from Western Europe, and more specifically its Alpine regions, where a significant percentage share of the general population is still employed in the primary sector, with a high ratio of self-employed farmers, and where the occupational health and safety preventive practices are strictly regulated [5,30,37-40].

The primary objective of this study was therefore to evaluate the relationship between high air temperature and OIs in AWs from the subalpine Autonomous Province of Trento (APT) during the time period 2000-2013. The secondary objective was similarly to retrospectively assess whether an increased rate of OIs is actually associated with HWs.

\section{MATERIAL AND METHODS \\ Settings}

Autonomous Province of Trento (APT) is located in Italy's North East, covers a total area of $6214 \mathrm{~km}^{2}\left(2399 \mathrm{mi}^{2}\right)$ and has a population of 537416 habitants (2015 census). The 
nature of its subalpine territory is overwhelmingly mountainous ( $20 \%$ is over $2000 \mathrm{~m}$ and $70 \%$ over $1000 \mathrm{~m}$ ), with forests covering around $50 \%$ of the total area: ultimately, the agricultural area only accounts for $22 \%$ of the total provincial territory, but in the last 2 centuries agriculture has become the cornerstone for the regional economic development. Agricultural activities are clustered in the lower areas, where weather conditions during summer may be very dangerous for the health of people, as the air temperature may even reach and pass $40^{\circ} \mathrm{C}$, the daily average temperature approximates $30^{\circ} \mathrm{C}$, and hot climate is frequently associated with high humidity levels and weak winds [37].

According to available labor force statistics, the agricultural sector in APT directly accounts for around 20000 employees: as available figures report the activity of 16446 agricultural farms including 11958 agricultural enterprises, usually of small extent $(89 \%$ are smaller than 5 ha and $56 \%$ smaller than 1 ha), most of AWs are either self-employed or hobby farmers. These figures however don't take into account seasonal and part-time employees, the number of whom may largely exceed full-time employees [37-40].

\section{Occupational injuries}

Data on all OIs occurred in the APT from 1 January 2000 to 31 December 2013 was retrieved through the institutional database of the National Insurance for Occupational Illness and Injuries (Istituto Nazionale Assicurazione Infortuni sul Lavoro - INAIL) "Information flow INAIL to Regions" ("Flussi Informativi INAIL Regioni”) [41]. The National Insurance for Occupational Illness and Injuries insurance is compulsory in the activities that the law defines as risky, and protects workers against damages due to work-related accidents and occupational diseases. Available data included any accident occurring at work and causing a trauma to one or more people. Even though only events causing more than 3 days of absence from work are usually compensated by the INAIL, origi- nal database included all events notified to the INAIL during the aforementioned time period. Data was preventively anonymized in order to include only economic sector of the worker, age at the time of the event, sex, and country of birth, and eventually incorporated reference to the geographical site (municipality-level detail) and calendar date of the events, the nature of injury, bodily location, mechanism, and agency of injury/disease.

In order to identify events that may have been associated with severe heat exposure both directly (e.g., heat illnesses, heat stroke, etc.) and indirectly, because of reduced vigilance and tolerance to physical efforts (e.g., trauma associated with falls; incidents including slipping, dropping tools, and errors of judgement), we applied a broad definition of events, by retrieving all injuries occurring during the warmer months of the year (i.e., May, June, July, August and September) including weekend and holidays, for subjects whose insurance status was identified as AWs, and excluding all cases:

- in which description defined as occurred on route, home to work place and vice versa,

- associated with animal bites or punctures,

- without information on the place of injury,

- those in the validation phase,

- in which available data did not include detailed information about factors of the working environment potentially modifying actual exposures to high temperatures (i.e., presence of heat sources, but also air conditioning or ventilation), also occupational injuries that clearly occurred indoors were similarly excluded.

\section{Meteorological data}

Meteorological data was retrieved from the Meteotrentino Service [42] of the APT for the time period from 1 January 2000 to 31 December 2013. Meteotrentino archive includes data from a total of 214 meteorological stations scattered over the provincial area, allowing to direct link geographical site of OIs, as defined 
at municipal level, with air temperature at the time of the accident. Available data included daily maximum temperature of the air $\left(\mathrm{T}_{\max }\right)$, and daily average air temperature $\left(\mathrm{T}_{\text {day }}\right)$. As exposure to severe heat has been associated with OIs through a non-linear association, with a presumptive role for cumulative, short-term exposure $[1,3,7,29,35], \mathrm{T}_{\text {day }}$ was assessed in 3 time lags, i.e., for the day of the event $\left(\mathrm{T}_{\text {day }} \operatorname{lag} 0\right)$, for the day of the event and during the previous $24 \mathrm{~h}\left(\mathrm{~T}_{\text {day }}\right.$ lag 1$)$, for the day of the event and during the previous $48 \mathrm{~h}\left(\mathrm{~T}_{\text {day }}\right.$ lag 2$)$.

In order to assess whether days with higher environmental temperature were actually associated with increased daily rates of OIs, the whole observation period was initially dichotomized as HW time period vs. non-HW time period. Currently, there is no universal definition of a HW, although it may be broadly defined as a prolonged period of excessive heat. In order to more easily compare our results with similar types of research, we have defined a HW event as a time period including at least 3 consecutive days with $\mathrm{T}_{\text {max }} \geq 35^{\circ} \mathrm{C}[28]$.

Moreover, collected data from the meteorological stations (i.e., $\mathrm{T}_{\max }, \mathrm{T}_{\text {day }}$ lag 0 to lag 2 ) allowed to categorize all calendar days into 3 exposure groups (i.e., days on which temperatures were $<75$ th percentile, from $75-95$ th percentile, higher than 95 th percentile).

\section{Ethics}

The study included only a retrospective assessment of data available through an Institutional Database, and the analysis was performed as a part of the official duties of the Occupational Health and Safety Unit (L'Unità Operativa di Prevenzione e Sicurezza negli Ambienti di Lavoro UOPSAL). Personal data was restricted to information about the OIs, and was treated in order to guarantee the respect of privacy of the involved workers, as specifically stated by Italian Law No. 675 of 1996 on personal data protection. Therefore, the study did not require preliminary evaluation by the local Ethical Committee.

\section{Statistical analysis}

Continuous variables were tested for normal distribution (D'Agostino and Pearson omnibus normality test): where the corresponding $\mathrm{p}$ value was $<0.10$, normality distribution was assumed as rejected and variables were compared through Mann-Whitney or Kruskal-Wallis test for multiple independent samples. On the other hand, variables passing the normality check (D'Agostino and Pearson $p \geq 0.10$ ) were compared using the Student's t-test or ANOVA, where appropriate.

Crude rates of injuries per day were calculated for the study period, by day of the week, by month, for HW vs. non-HW time periods, and eventually for the 3 exposure groups (i.e., $<75$ th percentile, $75-95$ th percentile, $>95$ th percentile), the latter assessed as $\mathrm{T}_{\text {max }}$ and $\mathrm{T}_{\text {day }}$ (lag 0 to lag 2). We assumed that the recorded events (i.e., OIs) were mutually independent, and although influenced by demographic factors and by the extent of agricultural activities performed in that time period, eventually related to air temperatures. In order to adjust crude rates for factors having a presumptive effect on the outcome variable injury rate, a Poisson regression model was implemented including environmental temperatures as the independent variable and age, sex, ethnicity (i.e., Italian born vs. nonItalian born people), job title (i.e., owner, employee full time, employee part time), progressive day of the week and calendar month as covariates [43]. The model did not include factors such as heat sources in the workplace, noise exposure, use of machineries, type of employment, etc., as not universally available from the reports. Similarly, we did not assess the physical effort at time of the events, as available data was inconsistently appropriate for such characterization. The daily injury rates reported for days comprised in the $<75$ th percentile groups $\left(\mathrm{T}_{\text {max }}, \mathrm{T}_{\text {day }}\right.$ lag 0 to lag 2) were defined as the referent ones.

Significance level was $p<0.05$ for all statistical tests.

All the analyses were performed in SPSS 24 (IBM Corp., Armonk, USA). 


\section{RESULTS}

\section{Meteorological data}

The average $\mathrm{T}_{\text {day }}$ during the period from 1 January 2000 to 31 December 2013 was $10.9 \pm 7.8^{\circ} \mathrm{C}$, with the corresponding $\mathrm{T}_{\text {day }}$ for warm season (1 May-30 September) being $18.4 \pm 3.7^{\circ} \mathrm{C}$ for lag $0,17.0 \pm 3.6^{\circ} \mathrm{C}$ for lag 1 , and $16.9 \pm 3.5^{\circ} \mathrm{C}$ for lag 2 . Highest daily temperatures were associated with the month of July $\left(\mathrm{T}_{\text {day }}\right.$ lag $\left.0=19.3 \pm 2.6^{\circ} \mathrm{C}\right)$ followed by August $\left(\mathrm{T}_{\text {day }}\right.$ lag $\left.0=19.0 \pm 2.8^{\circ} \mathrm{C}\right)$, June $\left(\mathrm{T}_{\text {day }}\right.$ lag $\left.0=17.9 \pm 3.2^{\circ} \mathrm{C}\right)$, September $\left(\mathrm{T}_{\text {day }}\right.$ lag $\left.0=14.6 \pm 2.8^{\circ} \mathrm{C}\right)$, being lower in May $\left(\mathrm{T}_{\text {day }} \operatorname{lag} 0=13.9 \pm 2.6^{\circ} \mathrm{C}\right)$. Focusing on $\mathrm{T}_{\text {max }}$, average value was $30.3 \pm 4.4^{\circ} \mathrm{C}$ May to September, being higher in July (mean \pm standard deviation $(\mathrm{M} \pm \mathrm{SD})=32.9 \pm 3.8^{\circ} \mathrm{C}$ ), followed by August $\left(\mathrm{M} \pm \mathrm{SD}=31.1 \pm 4.1^{\circ} \mathrm{C}\right)$, June $(\mathrm{M} \pm \mathrm{SD}$ $\left.=31.1 \pm 4.1^{\circ} \mathrm{C}\right)$, September $\left(\mathrm{M} \pm \mathrm{SD}=27.5 \pm 3.3^{\circ} \mathrm{C}\right)$ and eventually May $\left(\mathrm{M} \pm \mathrm{SD}=27.3 \pm 4 \cdot 1^{\circ} \mathrm{C}\right)$. Detailed categorization of $\mathrm{T}_{\text {day }}$ (lag 0 to lag 2 ) and $\mathrm{T}_{\text {max }}$ by exposure groups is presented in the Table 1 .

A total of 32 episodes fulfilled the working definition of HW events (i.e., $\geq 3$ consecutive days over $35^{\circ} \mathrm{C}$ ), for a total of 237 days $(11.1 \%$ of total observation period): the duration of individual HWs ranged from 3 to 17 days $(\mathrm{M} \pm \mathrm{SD}=7.2 \pm 4.4$ days), with an average $\mathrm{T}_{\text {day }}$ of $23.6 \pm 1.6^{\circ} \mathrm{C}$, respectively. Average $\mathrm{T}_{\text {max }}$ for the same periods were $22 \pm 8.9^{\circ} \mathrm{C}$, respectively.

\section{Occupational injuries}

During the study period a total of 7325 occupational injuries occurred from May to September, and males $(\mathrm{N}=6241)$ outnumbered females $(\mathrm{N}=1084)$ for 5.8 to 1 ratio. The involved subjects had a mean age of $45 \pm 13.7$ years old, and males were also significantly younger ( $44.6 \pm 0.2$ years) than females $(47.3 \pm 0.4$ years, $\mathrm{p}<0.001)$. Most of events involved prevalently farm owners $(63.1 \%)$, followed by full-time $(24.1 \%)$ and parttime employees (13.8\%). Overall, $11.5 \%$ of the subjects had a migrant background, with Romania (3.2\%), Po- land (1.6\%), Morocco and Albania (in both cases, 0.8\%) being the most representative countries of origin. In 15 cases, occupational accident resulted in the death of the worker $(0.2 \%)$ (Table 2$)$.

Estimated crude incidence rate for OIs was $3.4 \pm 2.3$ events/ day and, as shown in the Figure 1, Kruskal-Wallis H test revealed significant differences in the average daily rates
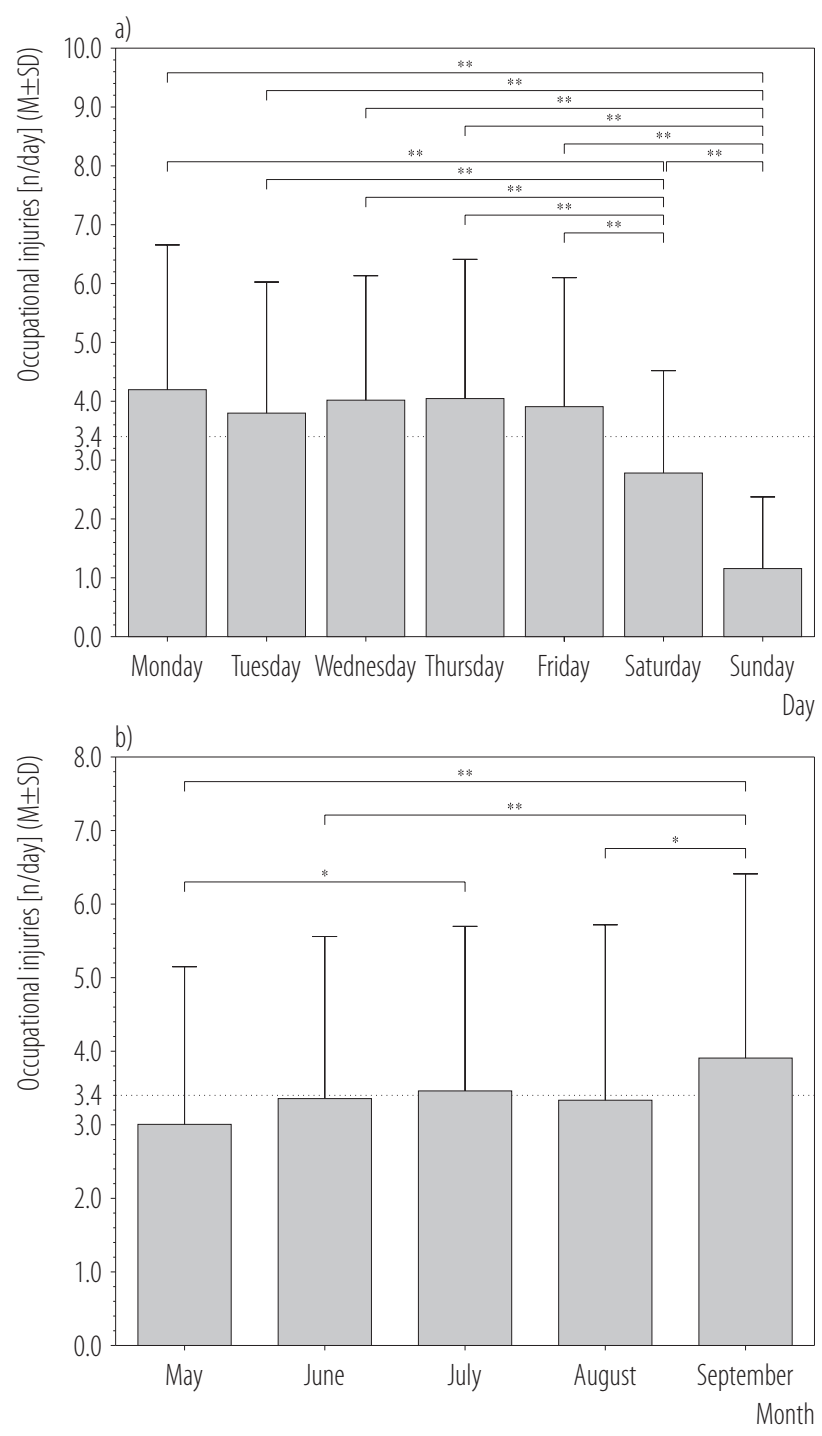

$\mathrm{M}$ - mean; SD - standard deviation.

$* \mathrm{p}<0.05 ; *$ p $<0.01$.

Fig. 1. Prevalence of occupational injuries in the agriculture in Autonomous Province of Trento, North-Eastern Italy, 2000-2013 (May-September), by: a) week day, b) calendar month 
Table 1. Air temperatures in Autonomous Province of Trento, North-Eastern Italy, 2000-2013 (May-September), by calendar month*

\begin{tabular}{|c|c|c|c|c|c|c|}
\hline \multirow{3}{*}{ Period } & \multicolumn{6}{|c|}{$\begin{array}{c}\text { Temperature } \\
{\left[{ }^{\circ} \mathrm{C}\right]} \\
\end{array}$} \\
\hline & \multirow{2}{*}{$\mathrm{M} \pm \mathrm{SD}$} & \multicolumn{5}{|c|}{ percentile } \\
\hline & & 5 th & 25th & $\mathrm{Me}$ & 75th & 95th \\
\hline \multicolumn{7}{|l|}{ May to September (total) } \\
\hline $\mathrm{T}_{\text {day }} \operatorname{lag} 0$ & $18.4 \pm 3.7$ & 12.2 & 15.8 & 18.5 & 21.3 & 24.1 \\
\hline $\mathrm{T}_{\text {day }}$ lag 1 & $17.0 \pm 3.6$ & 10.9 & 14.4 & 17.1 & 19.9 & 22.5 \\
\hline $\mathrm{T}_{\text {day }} \operatorname{lag} 2$ & $16.9 \pm 3.5$ & 11.0 & 14.4 & 17.0 & 19.7 & 22.3 \\
\hline $\mathrm{T}_{\max }$ & $30.3 \pm 4.4$ & 22.9 & 27.5 & 30.4 & 33.3 & 37.1 \\
\hline \multicolumn{7}{|l|}{ May } \\
\hline $\mathrm{T}_{\text {day }} \operatorname{lag} 0$ & $13.9 \pm 3.1$ & 10.6 & 13.2 & 15.1 & 17.4 & 20.5 \\
\hline $\mathrm{T}_{\text {day }}$ lag 1 & $13.9 \pm 2.9$ & 9.5 & 11.7 & 13.8 & 16.0 & 18.5 \\
\hline $\mathrm{T}_{\text {day }} \operatorname{lag} 2$ & $13.8 \pm 2.8$ & 9.7 & 11.8 & 13.7 & 15.8 & 18.6 \\
\hline $\mathrm{T}_{\max }$ & $27.3 \pm 4.1$ & 21.2 & 24.7 & 27.2 & 29.8 & 33.0 \\
\hline \multicolumn{7}{|l|}{ June } \\
\hline $\mathrm{T}_{\text {day }}$ lag 0 & $17.9 \pm 3.2$ & 13.8 & 17.0 & 19.2 & 22.0 & 24.2 \\
\hline $\mathrm{T}_{\text {day }} \operatorname{lag} 1$ & $17.8 \pm 3.1$ & 12.4 & 15.6 & 17.7 & 20.4 & 22.7 \\
\hline $\mathrm{T}_{\text {day }} \operatorname{lag} 2$ & $17.7 \pm 3.1$ & 12.8 & 15.6 & 17.6 & 20.4 & 22.6 \\
\hline $\mathrm{T}_{\max }$ & $31.1 \pm 4.1$ & 24.2 & 28.1 & 31.5 & 34.3 & 37.3 \\
\hline \multicolumn{7}{|l|}{ July } \\
\hline $\mathrm{T}_{\text {day }} \operatorname{lag} 0$ & $19.3 \pm 2.6$ & 16.0 & 19.0 & 21.3 & 22.6 & 24.8 \\
\hline $\mathrm{T}_{\text {day }}$ lag 1 & $19.3 \pm 2.7$ & 14.8 & 17.7 & 19.8 & 21.0 & 23.1 \\
\hline $\mathrm{T}_{\text {day }} \operatorname{lag} 2$ & $19.3 \pm 2.3$ & 15.0 & 17.7 & 17.7 & 21.0 & 23.0 \\
\hline $\mathrm{T}_{\max }$ & $32.9 \pm 3.8$ & 27.8 & 31.0 & 33.1 & 34.7 & 37.5 \\
\hline \multicolumn{7}{|l|}{ August } \\
\hline $\mathrm{T}_{\text {day }}$ lag 0 & $19.0 \pm 2.8$ & 15.6 & 18.9 & 20.6 & 22.4 & 25.1 \\
\hline $\mathrm{T}_{\text {day }} \operatorname{lag} 1$ & $19.1 \pm 2.7$ & 14.5 & 17.4 & 19.1 & 20.9 & 23.6 \\
\hline $\mathrm{T}_{\text {day }} \operatorname{lag} 2$ & $19.1 \pm 2.6$ & 14.9 & 17.5 & 19.1 & 20.9 & 23.5 \\
\hline $\mathrm{T}_{\max }$ & $32.3 \pm 3.8$ & 25.8 & 30.0 & 32.7 & 34.5 & 38.0 \\
\hline \multicolumn{7}{|l|}{ September } \\
\hline $\mathrm{T}_{\text {day }} \operatorname{lag} 0$ & $14.6 \pm 2.8$ & 11.0 & 14.4 & 16.0 & 17.7 & 20.8 \\
\hline $\mathrm{T}_{\text {day }}$ lag 1 & $14.7 \pm 2.7$ & 9.7 & 13.2 & 14.8 & 16.3 & 19.3 \\
\hline $\mathrm{T}_{\text {day }} \operatorname{lag} 2$ & $14.8 \pm 2.6$ & 9.9 & 134 & 14.8 & 16.3 & 19.3 \\
\hline $\mathrm{T}_{\max }$ & $27.5 \pm 3.3$ & 22.0 & 25.4 & 28.0 & 29.8 & 32.6 \\
\hline
\end{tabular}

$\mathrm{T}_{\mathrm{day}}$ - daily average air temperature; $\mathrm{T}_{\max }$ - daily maximum air temperature.

$* \mathrm{~T}_{\text {day }}$ was assessed for the day of the event (lag 0 ), for the day of the event and during the previous $24 \mathrm{~h}$ (lag 1 ), for the day of the event and during the previous $48 \mathrm{~h}$ (lag 2).

$\mathrm{M}$ - mean; SD - standard deviation; Me - median. 
Table 2. Study group characteristics and occupational injuries in the agriculture in Autonomous Province of Trento, North-Eastern Italy, 2000-2013 (May-September)

\begin{tabular}{|c|c|}
\hline Characteristics & $\begin{array}{c}\text { Respondents } \\
(\mathrm{N}=7325)\end{array}$ \\
\hline Occupational injuries crude rate [n/day] $(\mathrm{M} \pm \mathrm{SD})$ & $3.4 \pm 2.3$ \\
\hline Deaths $[\mathrm{n}(\%)]$ & $15(0.2)$ \\
\hline \multicolumn{2}{|l|}{ Gender $[\mathrm{n}(\%)]$} \\
\hline males & $6241(85.2)$ \\
\hline females & $1084(14.8)$ \\
\hline Age $^{a}$ [years] $(\mathrm{M} \pm \mathrm{SD})$ & $45.0 \pm 13.7$ \\
\hline \multicolumn{2}{|l|}{ Country of origin $[n(\%)]$} \\
\hline Italian born people & $6483(88.5)$ \\
\hline foreign born people & $842(11.5)$ \\
\hline Romania & $234(3.2)$ \\
\hline Poland & $117(1.6)$ \\
\hline Albania & $58(0.8)$ \\
\hline Morocco & $58(0.8)$ \\
\hline other & $467(6.4)$ \\
\hline \multicolumn{2}{|l|}{ Job titles $[\mathrm{n}(\%)]$} \\
\hline owners & $4622(63.1)$ \\
\hline \multicolumn{2}{|l|}{ employees } \\
\hline full time & $1692(23.1)$ \\
\hline part-time & $1011(13.8)$ \\
\hline
\end{tabular}

Explanations as in Table 1.

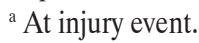

among the calendar months $(\mathrm{p}<0.0001)$, with lower rates in May $(\mathrm{M} \pm \mathrm{SD}=3 \pm 2.1$ events/day) and higher in September $(\mathrm{M} \pm \mathrm{SD}=3.9 \pm 2.5$ events/day $)$. During the working week, daily rates of OIs were also higher from Monday to Friday, and in particular on Monday $(\mathrm{M} \pm \mathrm{SD}=4.2 \pm 2.4$ events/day) $(\mathrm{p}<0.0001)$, being lower on Saturday $(\mathrm{M} \pm \mathrm{SD}=2.8 \pm 1.75$ events/day $)$ and on Sunday $(\mathrm{M} \pm \mathrm{SD}=1.2 \pm 1.2$ events/day). Crude daily rates of OIs were also higher during the HW events $(\mathrm{M} \pm \mathrm{SD}=3.7 \pm 2.5$ vs. $3.4 \pm 2.3$ events/day $)$ with a correspondent incidence rate ratio (IRR) equal to 1.09 (95\% confidence interval (CI): 1.02-1.17, $\mathrm{p}=0.0165)$.
The Table 3 shows the daily rates of OIs by exposure groups for $\mathrm{T}_{\text {day }}$ (lag 0 to lag 2) and $\mathrm{T}_{\text {max }}$, for the whole study period and by month. Focusing on the whole summer season (i.e., May to September), rates of OIs increased from < 75th $\left(\mathrm{M} \pm \mathrm{SD}=3.38 \pm 2.31\right.$ in $\mathrm{T}_{\text {day }}$ lag $0,3.38 \pm 2.33$ in $\mathrm{T}_{\text {day }}$ lag $1,3.39 \pm 2.33$ in $\mathrm{T}_{\text {day }}$ lag 2 , and $3.39 \pm 2.32$ in $\mathrm{T}_{\max }$ ) to $>95$ th percentile exposure groups $\left(\mathrm{M} \pm \mathrm{SD}=3.75 \pm 2.33\right.$ in $\mathrm{T}_{\text {day }}$ lag $0,3.80 \pm 2.52$ in $\mathrm{T}_{\text {day }}$ lag 1 , $3.72 \pm 2.37$ in $\mathrm{T}_{\text {day }}$ lag 2, and 3.70 \pm 2.73 in $\mathrm{T}_{\max }$ ) but Kruskal-Wallis $\mathrm{H}$ test revealed no significant differences among exposure groups ( $\mathrm{p}>0.05$ for all comparisons). Daily OIs rates exhibited a similar trend also when data was broken down by calendar months, as they increased 
Table 3. Prevalence of occupational injuries in the agriculture in Autonomous Province of Trento, North-Eastern Italy, 2000-2013 (May-September), by exposure groups as defined by maximum $\left(\mathrm{T}_{\text {max }}\right)$ and average $\left(\mathrm{T}_{\text {day }}\right)$ daily air temperatures*

\begin{tabular}{|c|c|c|c|c|}
\hline \multirow[t]{2}{*}{ Period } & \multicolumn{3}{|c|}{$\begin{array}{c}\text { Exposure groups injury rate } \\
{\left[\begin{array}{c}\mathrm{n} / \mathrm{day}] \\
(\mathrm{M} \pm \mathrm{SD})\end{array}\right.}\end{array}$} & \multirow[t]{2}{*}{$\mathrm{p}^{\mathrm{a}}$} \\
\hline & $<75$ th percentile & 75-95th percentile & $>95$ th percentile & \\
\hline \multicolumn{5}{|l|}{ May to September (total) } \\
\hline $\mathrm{T}_{\text {day }} \operatorname{lag} 0$ & $3.4 \pm 2.3$ & $3.5 \pm 2.2$ & $3.7 \pm 2.3$ & 0.338 \\
\hline $\mathrm{T}_{\text {day }} \operatorname{lag} 1$ & $3.4 \pm 2.3$ & $3.5 \pm 2.2$ & $3.8 \pm 2.5$ & 0.149 \\
\hline $\mathrm{T}_{\text {day }} \operatorname{lag} 2$ & $3.4 \pm 2.3$ & $3.4 \pm 2.2$ & $3.7 \pm 2.4$ & 0.230 \\
\hline $\mathrm{T}_{\max }$ & $3.4 \pm 2.3$ & $3.5 \pm 2.2$ & $3.7 \pm 2.7$ & 0.385 \\
\hline \multicolumn{5}{|l|}{ May } \\
\hline $\mathrm{T}_{\text {day }} \operatorname{lag} 0$ & $2.9 \pm 2.1$ & $3.4 \pm 2.2$ & $3.1 \pm 2.1$ & 0.117 \\
\hline $\mathrm{T}_{\text {day }} \operatorname{lag} 1$ & $2.9 \pm 2.1$ & $3.3 \pm 2.4$ & $2.9 \pm 2.0$ & 0.573 \\
\hline $\mathrm{T}_{\text {day }}$ lag 2 & $2.9 \pm 2.1$ & $3.3 \pm 2.2$ & $2.8 \pm 2.1$ & 0.411 \\
\hline $\mathrm{T}_{\max }$ & $2.8 \pm 2.1$ & $3.5 \pm 2.3$ & $3.3 \pm 2.3$ & 0.099 \\
\hline \multicolumn{5}{|l|}{ June } \\
\hline $\mathrm{T}_{\text {day }} \operatorname{lag} 0$ & $3.2 \pm 2.1$ & $3.7 \pm 2.4$ & $4.2 \pm 2.4$ & 0.065 \\
\hline $\mathrm{T}_{\text {day }} \operatorname{lag} 1$ & $3.2 \pm 2.1$ & $3.7 \pm 2.2$ & $4.8 \pm 2.5$ & 0.011 \\
\hline $\mathrm{T}_{\text {day }} \operatorname{lag} 2$ & $3.2 \pm 2.2$ & $3.5 \pm 2.1$ & $4.9 \pm 2.5$ & 0.015 \\
\hline $\mathrm{T}_{\max }$ & $3.2 \pm 2.1$ & $3.8 \pm 2.5$ & $3.9 \pm 2.2$ & 0.074 \\
\hline \multicolumn{5}{|l|}{ July } \\
\hline $\mathrm{T}_{\text {day }} \operatorname{lag} 0$ & $3.4 \pm 2.2$ & $3.7 \pm 2.3$ & $3.8 \pm 2.4$ & 0.374 \\
\hline $\mathrm{T}_{\text {day }} \operatorname{lag} 1$ & $3.5 \pm 2.2$ & $3.4 \pm 2.2$ & $3.5 \pm 2.8$ & 0.939 \\
\hline $\mathrm{T}_{\text {day }}$ lag 2 & $3.5 \pm 2.2$ & $3.7 \pm 2.5$ & $2.8 \pm 2.0$ & 0.345 \\
\hline $\mathrm{T}_{\max }$ & $3.5 \pm 2.2$ & $3.1 \pm 2.1$ & $3.7 \pm 2.9$ & 0.353 \\
\hline \multicolumn{5}{|l|}{ August } \\
\hline $\mathrm{T}_{\text {day }} \operatorname{lag} 0$ & $3.3 \pm 2.4$ & $3.4 \pm 2.2$ & $3.5 \pm 3.6$ & 0.661 \\
\hline $\mathrm{T}_{\mathrm{day}}$ lag 1 & $3.3 \pm 2.3$ & $3.6 \pm 2.7$ & $4.1 \pm 1.8$ & 0.122 \\
\hline $\mathrm{T}_{\text {day }} \operatorname{lag} 2$ & $3.2 \pm 2.3$ & $3.7 \pm 2.7$ & $3.8 \pm 1.8$ & 0.139 \\
\hline $\mathrm{T}_{\max }$ & $3.3 \pm 2.3$ & $3.5 \pm 2.7$ & $3.5 \pm 2.3$ & 0.905 \\
\hline \multicolumn{5}{|l|}{ September } \\
\hline $\mathrm{T}_{\text {day }} \operatorname{lag} 0$ & $2.5 \pm 1.6$ & $3.8 \pm 2.6$ & $4.1 \pm 2.5$ & 0.020 \\
\hline $\mathrm{T}_{\text {day }}$ lag 1 & $2.4 \pm 1.5$ & $3.9 \pm 2.6$ & $4.0 \pm 2.5$ & 0.010 \\
\hline $\mathrm{T}_{\text {day }}$ lag 2 & $2.6 \pm 1.4$ & $3.8 \pm 2.7$ & $4.1 \pm 2.5$ & 0.018 \\
\hline $\mathrm{T}_{\max }$ & $3.1 \pm 1.2$ & $3.9 \pm 2.5$ & $4.1 \pm 2.7$ & 0.032 \\
\hline
\end{tabular}

${ }^{a}$ In Kruskal-Wallis $\mathrm{H}$ test.

Abbreviations and explanations as in Table 1. 
Table 4. Risk of occupational injuries in the agriculture in Autonomous Province of Trento, North-Eastern Italy, 2000-2013 (May-September), by exposure groups as defined by maximum $\left(\mathrm{T}_{\max }\right)$ and average $\left(\mathrm{T}_{\text {day }}\right)$ daily air temperatures*

\begin{tabular}{|c|c|c|c|c|c|c|}
\hline \multirow{3}{*}{ Period } & \multicolumn{6}{|c|}{ Risk for exposure groups ${ }^{\mathrm{a}}$} \\
\hline & \multicolumn{3}{|c|}{ 75-95th percentile } & \multicolumn{3}{|c|}{$>95$ th percentile } \\
\hline & $\mathrm{p}$ & $\mathrm{OR}^{\mathrm{b}}$ & $95 \% \mathrm{CI}$ & $\mathrm{p}$ & $\mathrm{OR}^{\mathrm{b}}$ & $95 \% \mathrm{CI}$ \\
\hline \multicolumn{7}{|c|}{ May to September (total) } \\
\hline $\mathrm{T}_{\mathrm{day}} \operatorname{lag} 0$ & 0.565 & 1.019 & $0.957-1.085$ & 0.035 & 1.119 & $1.008-1.242$ \\
\hline $\mathrm{T}_{\text {day }} \operatorname{lag} 1$ & 0.446 & 1.025 & $0.962-1.091$ & 0.027 & 1.125 & $1.013-1.249$ \\
\hline $\mathrm{T}_{\text {day }} \operatorname{lag} 2$ & 0.699 & 1.013 & $0.950-1.079$ & 0.058 & 1.107 & $0.997-1.229$ \\
\hline $\mathrm{T}_{\max }$ & 0.555 & 1.019 & $0.956-1.089$ & 0.013 & 1.144 & $1.029-1.272$ \\
\hline \multicolumn{7}{|l|}{ May } \\
\hline $\mathrm{T}_{\text {day }} \operatorname{lag} 0$ & 0.962 & 1.006 & $0.791-1.279$ & 0.067 & 1.130 & $0.992-1.287$ \\
\hline $\mathrm{T}_{\text {day }} \operatorname{lag} 1$ & 0.203 & 1.094 & $0.953-1.256$ & 0.548 & 0.932 & $0.741-1.173$ \\
\hline $\mathrm{T}_{\text {day }} \operatorname{lag} 2$ & 0.070 & 1.131 & $0.990-1.291$ & 0.334 & 0.885 & $0.691-1.134$ \\
\hline $\mathrm{T}_{\max }$ & 0.441 & 1.097 & $0.867-1.388$ & 0.003 & 1.212 & $1.066-1.379$ \\
\hline \multicolumn{7}{|l|}{ June } \\
\hline $\mathrm{T}_{\text {day }} \operatorname{lag} 0$ & 0.062 & 1.131 & $0.994-1.288$ & 0.023 & 1.263 & $1.033-1.544$ \\
\hline $\mathrm{T}_{\text {day }} \operatorname{lag} 1$ & 0.115 & 1.107 & $0.976-1.257$ & 0.004 & 1.375 & $1.109-1.705$ \\
\hline $\mathrm{T}_{\text {day }}$ lag 2 & 0.478 & 1.048 & $0.921-1.191$ & 0.001 & 1.411 & $1.145-1.738$ \\
\hline $\mathrm{T}_{\max }$ & 0.032 & 1.149 & $1.012-1.304$ & 0.026 & 1.280 & $1.031-1.590$ \\
\hline \multicolumn{7}{|l|}{ July } \\
\hline $\mathrm{T}_{\text {day }} \operatorname{lag} 0$ & 0.297 & 1.073 & $0.940-1.225$ & 0.994 & 1.001 & $0.824-1.251$ \\
\hline $\mathrm{T}_{\text {day }} \operatorname{lag} 1$ & 0.585 & 0.964 & 0.846-1.099 & 0.558 & 1.072 & $0.850-1.352$ \\
\hline $\mathrm{T}_{\text {day }}$ lag 2 & 0.674 & 1.028 & $0.905-1.167$ & 0.496 & 0.909 & 0.689-1.197 \\
\hline $\mathrm{T}_{\max }$ & 0.069 & 0.885 & $0.776-1.010$ & 0.728 & 1.040 & $0.833-1.298$ \\
\hline \multicolumn{7}{|l|}{ August } \\
\hline $\mathrm{T}_{\text {day }} \operatorname{lag} 0$ & 0.599 & 1.035 & $0.911-1.175$ & 0.435 & 1.097 & $0.869-1.385$ \\
\hline $\mathrm{T}_{\text {day }}$ lag 1 & 0.294 & 1.070 & $0.943-1.214$ & 0.130 & 1.190 & $0.950-1.489$ \\
\hline $\mathrm{T}_{\text {day }}$ lag 2 & 0.485 & 1.079 & $0.972-1.336$ & 0.009 & 1.181 & $1.043-1.337$ \\
\hline $\mathrm{T}_{\max }$ & 0.393 & 1.057 & $0.931-1.199$ & 0.776 & 0.966 & $0.762-1.225$ \\
\hline \multicolumn{7}{|l|}{ September } \\
\hline $\mathrm{T}_{\text {day }} \operatorname{lag} 0$ & 0.014 & 1.441 & $1.076-1.982$ & 0.001 & 1.572 & $1.194-2.069$ \\
\hline $\mathrm{T}_{\text {day }}$ lag 1 & 0.004 & 1.550 & $1.151-2.086$ & 0.001 & 1.613 & $1.216-2.141$ \\
\hline $\mathrm{T}_{\text {day }} \operatorname{lag} 2$ & 0.014 & 1.414 & $1.072-1.864$ & 0.002 & 1.502 & $1.159-1.946$ \\
\hline $\mathrm{T}_{\max }$ & 0.016 & 1.363 & $1.060-1.752$ & 0.016 & 1.388 & $1.063-1.813$ \\
\hline
\end{tabular}

${ }^{a}$ The model included age, sex, ethnicity (i.e., Italian born vs. non-Italian born people), progressive day of the week and calendar month as covariates. ${ }^{\mathrm{b}}$ Odds ratio calculated through a Poisson regression analysis model, assuming the $<75$ th percentile groups as the referent ones. $\mathrm{OR}$ - odds ratio; $\mathrm{CI}$ - confidence interval.

Other explanations as in Table 1. 
from $<75$ th to $>95$ th percentile exposure group: a notable exception was represented by the month of May, the OIs peak of which was associated with the intermediate exposure group 75-95th percentile for both $\mathrm{T}_{\text {max }}$ and $\mathrm{T}_{\text {day }}$ (all time lags). However, differences were statistically significant only for the month of September, all the assessments $\left(\mathrm{T}_{\text {day }}\right.$ lag 0 to lag $2, \mathrm{~T}_{\max }: \mathrm{p}=0.020,0.010$, 0.018 and 0.032 , respectively), and for the month of June, relatively to $\mathrm{T}_{\text {day }} \operatorname{lag} 1(\mathrm{p}=0.011)$ and $\operatorname{lag} 2(\mathrm{p}=0.015)$.

Assuming the rates of OIs occurring on days on which $\mathrm{T}_{\text {day }}$ (lag 0 to lag 2) was comprised in the $<75$ th percentile exposure groups as the referent ones, the Poisson regression analysis modeled for the May-September time period identified significantly increased risk of OIs $(\mathrm{OR}=1.119,95 \% \mathrm{CI}$ : $1.008-1.242$, and $\mathrm{OR}=1.125,95 \%$ CI: 1.013-1.249) for the $>$ 95th percentile exposure groups as assessed in terms of $\mathrm{T}_{\text {day }}$ lag 0 and lag 1 whereas no significant differences were found for $\mathrm{T}_{\text {day }}$ lag 2. Similarly, days on which $\mathrm{T}_{\text {max }}$ was comprised in the $>95$ th percentile exposure group was associated with OIs rates $(\mathrm{OR}=1.144,95 \% \mathrm{CI}: 1.029-1.272)$ significantly greater than that found in the exposure groups $\mathrm{T}_{\max }<75$ th percentile and 75-95th percentile (Table 4). Focusing on the single calendar months, in the month of September days on which $\mathrm{T}_{\text {day }}$ (lag 0 to lag 2) and $\mathrm{T}_{\text {max }}$ were comprised in the exposure groups 75-95th percentile and $>95$ th percentile, the figures were associated with a risk for OIs significantly greater than the referent exposure groups $<75$ th percentile. A similarly increased risk for OIs was also found for the days comprised in the $>95$ th exposure groups for the months of June $\left(\mathrm{T}_{\max }\right.$ and $\mathrm{T}_{\text {day }}$, lag 0 to lag 2), August ( $\mathrm{T}_{\text {day }}$ lag 2) and May $\left(\mathrm{T}_{\max }\right)$ whereas no differences were found between reference groups and 75-95th exposure groups.

\section{DISCUSSION}

Our results show that both high daily temperatures and HWs events occurring during the warm season might be associated with increased incidence of OIs in the agricultural settings. Our study is therefore consistent with previous reports suggesting that hot weather conditions might represent a significant risk factor for work-related injuries [3,25,27-29,35]. A somehow increased risk for occupational injuries was then reported on days having highest $\mathrm{T}_{\text {day }}$ (lag 0 and lag 1 ) and $\mathrm{T}_{\text {max }}$ (i.e., > 95th percentile). Our results are therefore somehow contradictory with previous studies, in which an "inverted U"-shaped curve relationship was rather suggested. In other words, previous evidence suggests that the highest risk for work-related accidents would be reported on days characterized by severe but not extreme thermal conditions whereas days associated with either extreme hot or cold weather would have lower incidence rates [29,35].

Such evidences are usually explained through behavioral adaptations to severe climates. In other words, workers would avoid most strenuous activities during the hottest days and/or the hottest hours of the working day during the warm season and, similarly, coldest days of the cold season would be perceived as inappropriate to perform outdoor activities $[25,35]$. Furthermore, it should be recalled that in most high-income countries, hottest months of the warm season are associated with holidays, and vacations may further contribute to the reduced number of reported events, particularly in salaried workers [3,25,28,29,35].

These inconsistencies with available literature may be explained recalling some specificities of the agricultural activities in the Subalpine regions.

First of all, most of agricultural works follow crop growth $[5,19,44]$. Even though certain agricultural activities may benefit from a short delay when weather conditions are particularly uncomfortable, the majority of agricultural activities have to be performed in a restricted time window, following the timetable imposed by crops and cultivations, not allowing the AWs to usually spare holidays and weekend. Also during the warm season, the timing of irrigation and/or spraying of pesticides is not 
always very flexible and a series of hot, warm days with reduced rainfalls increase the requirements for artificial irrigation, forcing AWs to perform their activities even during HWs [30,33,34,45-47].

Not coincidentally, in our survey the daily average rate of OIs was similar from June to August, being higher in the calendar months of September and June, with the significant lack of effect for the central months of July and August, otherwise characterized by higher temperatures. Actually, APT is characterized by a reduced number of diverse cultures, mainly apples and vineyards, and pesticide application is usually clustered in the calendar months of June and in the second half of August $[3,4,9,27,35,47,48]$. The central weeks of summer season are therefore associated with a significant contraction of both workforce, with a substantial shrinkage of its more unskilled component and workload $[3,25,28,29,35]$. On the contrary, most of the agricultural activities are performed during the month of September, as it is associated with the harvest of grapes and the picking of fruits, and in the mountainous Alpine and Subalpine regions such as APT, mild or even warm air temperatures are not unusual $[37,39,45]$. In other words, the increased risk for OIs during the months of June and September, as well as the lack of effect for the calendar months of July, may be explained in terms of workload and workforce $[39,40,45,47]$.

In this regard, it should be stressed that, although temporary workers are usually associated with an increased risk for OIs $[39,40,45,47]$, the majority of the events included in our survey were reported from self-employed AWs. Despite the paucity of available data [46], evidence suggests that self-employed AWs would be at somehow increased risk for OIs, as they not only would personally perform more dangerous procedures (e.g., pesticide dispersal, use of transportation vehicles, etc.) but they also more frequently avoid appropriate preventive measures notwithstanding an appropriate awareness of related health and safety risks because of personal/economic interests [48-50]. In our survey the rates of OIs during weekends were again decreased but not abolished, and even though HWs events should have discouraged agricultural activities, in particular by salaried AWs, we identified a significantly increased incidence of OIs for this specific time frame [3,25,28,29,35].

Several limitation of our study has to be addressed.

Firstly, as data about air humidity, wind speed and solar irradiation was not available for all meteorological stations, only air temperature measures were included in the analysis, and the measurement of air temperature without taking into account broader weather conditions such as radiant heat, air humidity, wind speed and solar irradiation, should be assumed as a rough proxy of the actual exposure $[3,25,29,35]$. In this regard, it should be stressed that, in a mountainous region such as APT, air temperatures and also other climate factors significantly associated with heat-related health effects such as air humidity and solar irradiation may strikingly fluctuate over a restricted area because of the altitude [31,47,51]. In other terms, the assessment of air temperature at municipality level may also be affected by some inaccuracies [29,35]. However, as available data about air humidity, wind speed and solar irradiation was more diffusely scattered over the area of APT, their inclusion in the exposure assessment would have probably increased its inaccuracy.

Secondly, the data regarding the OIs was also affected by some inaccuracies. Available information about occupational injuries was retrieved from an institutional database, the reliability of which may be intrinsically questioned because of reporting bias. Moreover, database did not include accurate description of environmental (e.g., heat sources, extent of exposure to solar radiation, etc.) and individual risk factors for heat-related health effects such as the level of physical activity performed at the time of the event, as wells as the PPE possibly worn at time of the injury, hydration status, drug assumed before the event, etc. [47,52]. 
Thirdly, we lacked data about the total number of subjects employed in agricultural activities at the time of recorded accidents. In other terms, it is possible that our assessment ultimately underestimates the actual rates of OIs during the hottest days, at the same time overestimating them when workforce significantly inflates, as during wine harvesting and fruit picking [37-40,47,53,54].

\section{CONCLUSIONS}

In conclusion, this study confirms previous evidences suggesting that working outdoors with increased air temperatures and during HWs is associated with increased daily rates of occupational injuries. In light of available climate projections, our results are also of valuable interest for policymakers, as they ultimately suggest the opportunity to develop appropriate regulation and guidelines aimed to prevent exposure of AWs to highest air temperatures during the warm season. Even though avoiding agricultural activities during hottest days of the summer season could be acknowledged as a cost-effective preventive measure, the high number of occupational injuries reported among self-employed AWs, during holidays and even during HWs suggest that it may be limitedly effective.

\section{REFERENCES}

1. McInnes JA, Akram M, MacFarlane EM, Keegel T, Sim MR, Smith P. Association between high ambient temperature and acute work-related injury: A case-crossover analysis using workers' compensation claims data. Scand J Work Environ Health. 2017;43(1):86-94, https://doi.org/10.5271/sjweh.3602.

2. Kjellstrom T, Holmer I, Lemke B. Workplace heat stress, health and productivity - An increasing challenge for low and middle-income countries during climate change. Glob Health Action. 2009;2(1), https://doi.org/10.3402/gha.v2i0.2047.

3. Xiang J, Bi P, Pisaniello D, Hansen A. Health impacts of workplace heat exposure: An epidemiological review. Ind Health. 2014;52(2):91-101, https://doi.org/10.2486/indhealth. 2012-0145.
4. Semenza JC, McCullough JE, Flanders WD, McGeehin MA, Lumpkin JR. Excess hospital admissions during the July 1995 heat wave in Chicago. Am J Prev Med. 1999;16(4):269-77, https://doi.org/10.1016/S0749-3797(99) 00025-2.

5. Gubernot DM, Anderson GB, Hunting KL. Characterizing occupational heat-related mortality in the United States, 2000-2010: An analysis using the census of fatal occupational injuries database. Am J Ind Med. 2015;58(2):203-11, https://doi.org/10.1002/ajim.22381.

6. Madrigano J, Ito K, Johnson S, Kinney PL, Matte T. A caseonly study of vulnerability to heat wave-related mortality in New York City (2000-2011). Environ Health Perspect. 2015;123(7):672-8, https://doi.org/10.1289/ehp.1408178.

7. Lin Y-K, Chang C-K, Wang Y-C, Ho T-J. Acute and prolonged adverse effects of temperature on mortality from cardiovascular diseases. PLoS ONE. 2013;8(12):e82678, https://doi.org/10.1371/journal.pone.0082678.

8. Li Y, Cheng Y, Cui G, Peng C, Xu Y, Wang Y, et al. Association between high temperature and mortality in metropolitan areas of four cities in various climatic zones in China: A time-series study. Environ Health. 2014;13:65, https://doi.org/10.1186/1476-069X-13-65.

9. Bobb JF, Obermeyer Z, Wang Y, Dominici F. Cause-specific risk of hospital admission related to extreme heat in older adults. JAMA. 2014;312(24):2659-67, https://doi.org/ 10.1001/jama.2014.15715.

10. Harlan S, Chowell G, Yang S, Petitti DB, Morales Butler EJ, Ruddell BL, et al. Heat-related deaths in hot cities: Estimates of human tolerance to high temperature thresholds. Int J Environ Res Public Health. 2014;11(3):3304-26, https://doi.org/10.3390/ijerph110303304.

11. Semenza JC, Rubin CH, Falter KH, Selanikio JD, Flanders WD, Howe HL, et al. Heat-related deaths during the July 1995 heat wave in Chicago. N Eng J Med. 1996;335(2): 84-90, https://doi.org/10.1056/NEJM199607113350203.

12. Åström DO, Forsberg B, Rocklöv J. Heat wave impact on morbidity and mortality in the elderly population: A review 
of recent studies. Maturitas. 2011;69(2):99-105, https://doi. org/10.1016/j.maturitas.2011.03.008.

13. Stoecklin-Marois M, Hennessy-Burt T, Mitchell D, Schenker M. Heat-related illness knowledge and practices among California hired farm workers in the MICASA Study. Ind Health. 2013;51(1):47-55, https://doi.org/10.2486/indhealth. 2012-0128.

14. Xiang J, Hansen A, Pisaniello D, Bi P. Workers' perceptions of climate change related extreme heat exposure in South Australia: A cross-sectional survey. BMC Public Health. 2016;16:549, https://doi.org/10.1186/s12889-016-3241-4.

15. Majumder J, Bagepally B, Shah P, Kotadiya S, Yadav S, Naha N. Comparison of workers' perceptions toward work climate and health symptoms between ceramic and iron foundry workers. Indian J Occup Environ Med. 2016;20(1): 48-53, https://doi.org/10.4103/0019-5278.183845.

16. Kjellstrom T, Sawada S-I, Bernard TE, Parsons K, Rintamäki H, Holmér I. Climate change and occupational heat problems. Ind Health. 2013;51(1):1-2, https://doi.org/10.2486/ indhealth.MS5101ED.

17. Kjellstrom T, Briggs D, Freyberg C, Lemke B, Otto M, Hyatt $\mathrm{O}$. Heat, human performance, and occupational health: A key issue for the assessment of global climate change impacts. Ann Rev Public Health. 2016;37:97-112, https://doi. org/10.1146/annurev-publhealth-032315-021740.

18. Zhou L, Xin Z, Bai L, Wan F, Wang Y, Sang S, et al. Perceptions of heat risk to health: A qualitative study of professional bus drivers and their managers in Jinan, China. Int J Environ Res Public Health. 2014;11(2):1520-35, https:// doi.org/10.3390/ijerph110201520.

19. Adam-Poupart A, Smargiassi A, Busque M-A, Duguay P, Fournier M, Zayed J, et al. Effect of summer outdoor temperatures on work-related injuries in Quebec (Canada). Occup Environ Med. 2015;72(5):338-45, https://doi. org/10.1136/oemed-2014-102428.

20. Jay O, Kenny GP. Heat exposure in the Canadian workplace. Am J Ind Med. 2010;53(8):842-53, https://doi.org/10.1002/ ajim.20827.
21. Donoghue AM. Heat illness in the U.S. mining industry. Am J Ind Med. 2004;45(4):351-6, https://doi.org/10.1002/ ajim.10345.

22. Donoghue AM, Sinclair MJ, Bates GP. Heat exhaustion in a deep underground metalliferous mine. Occup Environ Med. 2000;57(3):165-74, https://doi.org/10.1136/oem.57.3.165.

23. Maeda T, Kaneko S-Y, Ohta M, Tanaka K, Sasaki A, Fukushima T. Risk factors for heatstroke among Japanese forestry workers. J Occup Health. 2006;48(4):223-9, https://doi. org/10.1539/joh.48.223.

24. Petitti DB, Harlan SL, Chowell-Puente G, Ruddell D. Occupation and environmental heat-associated deaths in Maricopa County, Arizona: A case-control study. PLoS ONE. 2013;8(5):e62596, https://doi.org/10.1371/journal.pone. 0062596.

25. Otte im Kampe E, Kovats S, Hajat S. Impact of high ambient temperature on unintentional injuries in high-income countries: A narrative systematic literature review. BMJ Open. 2016;6(2):e010399, https://doi.org/10.1136/bmjopen2015-010399.

26. Tawatsupa B, Lim LL-Y, Kjellstrom T, Seubsman S-A, Sleigh A; the Thai Cohort Study Team. Association between occupational heat stress and kidney disease among 37816 workers in the Thai Cohort Study (TCS). J Epidemiol. 2012;22(3):251-60, https://doi.org/10.2188/jea.JE20110082.

27. Xiang J, Hansen A, Pisaniello D, Bi P. Extreme heat and occupational heat illnesses in South Australia, 2001-2010. Occup Environ Med. 2015;72(8):580-6, https://doi.org/10.1136/ oemed-2014-102706.

28. Xiang J, Bi P, Pisaniello D, Hansen A. The impact of heatwaves on workers' health and safety in Adelaide, South Australia. Environ Res. 2014;133:90-5, https://doi.org/10.1016/ j.envres.2014.04.042.

29. Morabito M, Cecchi L, Crisci A, Modesti PA, Orlandini S. Relationship between work-related accidents and hot weather conditions in Tuscany (Central Italy). Ind Health. 2006;44(3):458-64, https://doi.org/10.2486/indhealth.44.458. 
30. Centers for Disease Control and Prevention. Heat related deaths among crop workers United States, 1992-2006. MMWR Morb Mortal Wkly Rep. 2008;57(24):649-53.

31. Zhang K, Arauz RF, Chen T-H, Cooper SP. Heat effects among migrant and seasonal farmworkers: A case study in Colorado. Occup Environ Med. 2016;73(5):324-8, https:// doi.org/10.1136/oemed-2015-103332.

32. Gubernot DM, Anderson GB, Hunting KL. The epidemiology of occupational heat exposure in the United States: A review of the literature and assessment of research needs in a changing climate. Int J Biometeorol. 2014;58(8): 1779-88, https://doi.org/10.1007/s00484-013-0752-x.

33. Mirabelli MC, Quandt SA, Crain R, Grzywacz JG, Robinson EN, Vallejos QM, et al. Symptoms of heat illness among Latino farm workers in North Carolina. Am J Prev Med. 2010;39(5):468-71, https://doi.org/10.1016/j.amepre. 2010.07.008.

34. Bethel J, Harger R. Heat-related illness among Oregon farmworkers. Int J Environ Res Public Health. 2014;11(9): 9273-85, https://doi.org/10.3390/ijerph110909273.

35. Morabito M, Iannuccilli M, Crisci A, Capecchi V, Baldasseroni A, Orlandini S, et al. Air temperature exposure and outdoor occupational injuries: A significant cold effect in Central Italy. Occup Environ Med. 2014;71(10):713-6, https://doi.org//10.1136/oemed-2014-102204.

36. Manzoli L, Sotgiu G, Magnavita N, Durando P; National Working Group on Occupational Hygiene of the Italian Society of Hygiene Preventive Medicine and Public Health (SItI). Evidence-based approach for continuous improvement of occupational health. Epidemiol Prev. 2015;39 (4 Suppl 1):81-5.

37. European Parliament, Directorate General for Internal Policies of the Union; Policy Department, Structural and Cohesion Policies. Agriculture in the Alpine Areas of Austria and Italy [Internet]. Brussels: The Parliament; 2007 [cited 2017 Jan 2]. Available from: http://www.europarl.europa.eu/ RegData/etudes/etudes/join/2007/389589/IPOL-AGRI_ ET(2007)389589_EN.pdf.
38. Agnolin C, Ioriatti C, Pontalti M, Venturelli MB. IFP experiences in Trentino, Italy. Acta Hortic. 2000;525:45-50, https://doi.org/10.17660/ActaHortic.2000.525.3.

39. Fabbris T, Michielin F. The economy of the Italian regions: Recent developments and responses to the economic crisis [Internet]. European Union Regional Policy; 2010 [cited 2017 Jan 2]. Available from: http://ec.europa.eu/regional_ policy/sources/docgener/work/2010_01_italian.pdf.

40. Bruno Kessler Foundation, The Research Institute for the Evaluation of Public Policies. [Report on the Social and Economic Status of Trentino Region, Edition 2014] [Internet]. Trento: The Institute; 2014 [cited 2017 Jan 2]. Available from: http://www.giunta.provincia.tn.it/binary/pat_giunta_09/XV_ legislatura/FBK_IRVAPP_Rapporto_situazione_economica_ sociale_del_Trentino_2014.1417771778.pdf. Italian.

41. Madeo G, Giaimo M. [Accidents at work in construction workers: A peculiar utilization of the information flow INAIL-ISPESL-Regions]. G Ital Med Lav Ergon. 2007;29(S3):632-4. Italian.

42. Meteotrentino [Internet]. Trento: Protezione Civile; c2017 [cited 2017 April 10] [Archives of meteorological data]. Available from: http:/www.meteotrentino.it/dati-meteo/ info-dati.aspx?id=218. Italian.

43. Bailer AJ, Reed LD, Stayner LT. Modeling fatal injury rates using Poisson regression: A case study of workers in agriculture, forestry, and fishing. J Saf Res. 1997;28(3):177-86, https://doi.org/10.1016/S0022-4375(97)80006-0.

44. Li J, Xu X, Ding G, Zhao Y, Zhao R, Xue F, et al. A cross-sectional study of heat wave-related knowledge, attitude, and practice among the public in the Licheng District of Jinan City, China. Int J Environ Res Public Health. 2016;13(7):648, https://doi.org/10.3390/ijerph13070648.

45. Organisation for Economic Co-operation and Development (OECD). The co-operative model in Trentino - Italy. A case study. Report [Internet]. Paris: The Organisation; 2014 [cited 2017 Jan 2]. Available from: https://www.oecd. org/cfe/leed/150202 The cooperative model in Trentino_FINAL with covers.pdf. 
46. Fleischer NL, Tiesman HM, Sumitani J, Mize T, Amarnath KK, Bayakly AR, et al. Public health impact of heat-related illness among migrant farmworkers. Am J Prev Med. 2013; 44(3):199-206, https://doi.org/10.1016/j.amepre.2012.10.020.

47. Riccò M, Razio B, Poletti L, Panato C. Knowledge, attitudes, and sun-safety practices among agricultural workers in the Autonomous Province of Trento, North-Eastern Italy (2016). G Ital Dermatol Venereol. Forthcoming 2017, https://doi.org/10.23736/S0392-0488.17.05672-3.

48. Nelson NG, Collins CL, Comstock RD, McKenzie LB. Exertional heat-related injuries treated in emergency departments in the U.S., 1997-2006. Am J Prev Med. 2011;40(1): 54-60, https://doi.org/10.1016/j.amepre.2010.09.031.

49. Karttunen J, Rautiainen RH. Distribution and characteristics of occupational injuries and diseases among farmers: A retrospective analysis of workers' compensation claims. Am J Ind Med. 2013;56(8):856-69, https://doi.org/10.1002/ ajim.22194.

50. Sam KG, Andrade HH, Pradhan L, Pradhan A, Sones SJ, Rao PGM, et al. Effectiveness of an educational program to promote pesticide safety among pesticide handlers of
South India. Int Arch Occup Environ Health. 2008;81(6): 787-95, https://doi.org/10.1007/s00420-007-0263-3.

51. Milon A, Sottas P-E, Bulliard J-L, Vernez D. Effective exposure to solar UV in building workers: Influence of local and individual factors. J Expo Sci Environ Epidemiol. 2007;17(1):58-68, https://doi.org/10.1038/sj.jes.7500521.

52. Bena A, Pasqualini O, Agnesi R, Baldasseroni A. [How to assess the effects of interventions for preventing work injuries? Observations on INAIL-ISPESL-Regioni indicators]. Epidemiol Prev. 2008;32(3):168-75. Italian.

53. European Commission, DG Agriculture and Rural Development, Unit Economic Analysis of EU Agriculture. How many people work in agriculture in the European Union? [Internet]. European Commission; 2013 [cited 2017 Jan 2]. Available from: http://ec.europa.eu/agriculture/sites/agriculture/files/rural-area-economics/briefs/pdf/08_en.pdf.

54. The Labour Agency of the Autonomous Province of Trento. Local job creation: How employment and training agencies can help [Internet]. Paris: OECD; 2012 [cited 2017 Jan 2]. Available from: https://www.oecd.org/cfe/leed/Report Local Job Creation Labour Agency Trento Italy.pdf.

This work is available in Open Access model and licensed under a Creative Commons Attribution-NonCommercial 3.0 Poland License - http://creativecommons.org/ licenses/by-nc/3.0/pl/deed.en. 